\title{
Popkultur im Fernsehen - Fernsehen in der Popkultur
}

\author{
Eine Einleitung
}

Stefan Greif, Nils Lehnert und AnnA-CARINA MeYWIRTh

Die stärkste aller populären Mächte
aber ist der Bildschirm [...].
STEENBLOCK (2010)

Abgesehen von den MTV- und VIVA-Clips gehört Popkultur im Fernsehen zu den ebenso wenig erforschten Aspekten der TV-Geschichte der letzten 60 Jahre wie die Erforschung und Reflexion des Fernsehens aus popästhetischer Perspektive. Dazu mag beigetragen haben, dass sich Fernsehen und Pop hierzulande fast zeitgleich etablieren und beide fortan als >Billigmedien $<$ gelten, die sich gegen avanciertere und (vermeintlich) >anwenderbezogenere $<$ Medien wie das Radio oder (später) das Internet nicht zu behaupten vermögen. Doch die noch heute populäre Gleichung, Pop und TV seien jeweils >Mainstream<, das Internet hingegen - bis NSA jedenfalls - ein avantgardistischer Freiraum, greift ebenfalls zu kurz, denn seit den 1990er Jahren nutzen mediensozialisierte und medienversierte (Pop-)Literaten und Künstler das Fernsehen vermehrt für eigene Inszenierungsstrategien, die ganz bewusst an teils bildungsaffine, teils popkulturelle Formen der Kunstvermittlung anknüpfen, diese gezielt in ihrer jeweiligen Überholtheit bloßstellen oder sie im Dienste transmedialer Verbreitungsformen weiterentwickeln. Darüber hinaus wird das TV in der Popkultur in unterschiedlichster Weise thematisiert, reflektiert und kritisiert und beispielsweise in Form von Installationen zum Gegenstand der Kunst. Während kulturkonservative Kritiker in dieser Annäherung eine Bestätigung ihrer Vorbehalte dem Pop und dem Fernsehen gegenüber sehen, verbindet sich mit ihr der Anspruch, die ohnehin marode $>$ Hochkultur mit ihren eigenen Waffen zu schlagen und via TV durch eine Pop- 
kultur zu ersetzen. Wie das Beispiel von Andy Warhol oder Rainald Goetz dokumentiert, ist mit dieser Infragestellung tradierter Deutungshegemonien weder die Stiftung neuer kultureller Direktiven noch eine Affirmation der reinen Spaßgesellschaft intendiert.

Pop, so ließe sich thesenartig zusammenfassen, erhebt hiermit den Anspruch, das TV als sinnstiftende Instanz ernster zu nehmen, solange es ein größeres, heterogeneres und bisweilen irritierend nonkonformeres Publikum erreicht als die kanonisierten Künste. Lässt man diese Überlegung gelten, mag auch das gewollt janusköpfige Konzept des Sammelbands nachvollziehbarer werden. So wird nämlich einerseits der Frage nachgegangen, wie sich das Fernsehen seit den 1960er Jahren mit der Popkultur auseinandersetzt, in welchen Genres das zunächst geschieht und mit welchen >Spielarten< des Pop - Beat, Rock, Slam, Siebdrucke, die Suppendosen zeigen, usw. - sich das TV angesichts einer sich zur Eventkultur entwickelnden Gesellschaft vorzugsweise arrangiert. Andererseits ist zu fragen, seit wann das Fernsehen Stilelemente der Popkultur (Serialisierung, Oberflächenästhetik, Starkult) adaptiert oder bereits in den 1950er Jahren vorprägt, ob Pop (Pop Art, Musik, Literatur) im TV nur in >populärer < Form präsentiert und rezipiert wird und schließlich, wie sich das Fernsehen mit seinen medialen Mechanismen und Formaten in der Popkultur und ihren künstlerischen Artefakten spiegelt.

\section{Popbegriff}

Um diese facettenreichen Vorhaben in einem Sammelband vereinen zu können, dessen Ziel es ist, ebenso facettenreich wahrgenommen zu werden, erscheint es widersprüchlich, einen gemeinsamen Nenner auszuformulieren, auf welchen immer wieder rekurriert werden sollte - insbesondere, wenn es dabei um Pop mit all seinen Komposita und Konnotationen geht. An die Stelle einer enggeführten Definition soll aus diesem Grund ein breit angelegtes Deutungs- und Verständnisspektrum des Wortfelds >Pop< treten und auch nicht explizit zwischen Pop und dem Populären unterschieden werden, wenngleich die Herausgeber die Einschätzung teilen, dass nicht alles Populäre zum Pop gehört, aber durch eine popästhetische Rezeptionsweise sehr wohl dazu werden kann. Bewusst lässt dieser Band daher eine >Definitionsoffenheit< zu, um in dem großen Rahmen >Popkultur und Fernsehen $<$ möglichst viele Perspektiven (mediale, historische, ästhetische etc.) zu versammeln. Dabei besteht das Potenzial forschungsseitig darin, der kreativen, vielschichtigen Auseinandersetzung mit Pop keinen Riegel vorzuschieben, gleichzeitig wird rezeptionsseitig die Diversität 
des Begriffs erfahrbar gemacht. Das Risiko, das mit einem solch breiten Definitionsspektrum einhergehen kann, besteht möglicherweise in dem Vorwurf der Nivellierung oder der Beliebigkeit der Begriffe. An dieser Stelle sei jedoch angemerkt, dass Offenheit nicht gleichbedeutend mit einer »willkürliche[n] Setzung« des Popbegriffs ist, sondern vielmehr »einem Gegenstand « gerecht werden soll, »der nicht als fest konturiertes materielles Ding vorliegt, sondern als Hervorbringung unterschiedlicher weltanschaulicher und ästhetischer Reden und Interessen jeweils neu oder anders gebildet wird « (Hecken 2009: 14). Die benannte Skala der möglichen Konzepte reicht dabei von Pop »give[s] the people what they want « (Harron, zit. n. Hecken 2012) bis hin zu einer dezidierten Trennung von E- und U-Kultur, von Pop ist »designed for a mass of people« (Hamilton, zit. n. Hecken 2009: 12) bis zu der Kritik an Pops Oberflächlichkeit. So erscheint die Lösung durchaus naheliegend, von Pop als >Prinzip <, >System<, >Diskurs< (Goer/Greif/Jacke 2013), >Phänomen<, >Konzept < oder >Archiv< (Baßler 2002) zu sprechen. Nach Diedrich Diederichsen dürfen solche Zuschreibungen freilich nicht übersehen lassen, dass Pop selbst permanenten Transformationen, das heißt der Neukodierung von bestehendem Material, unterworfen bleibt und infolgedessen eine hohe Dynamik aufweist (vgl. Diederichsen 1996: 38f.). Das einzig Beständige an Pop ist also sein Wandel.

All diesen Erklärungsansätzen muss insofern bewusst bleiben, dass Pop nicht als etwas Isoliertes oder Singuläres auftreten kann, sondern immer mit Kontexten verknüpft ist, sich dazu positioniert und gleichzeitig Stellungnahmen einfordert, sei es durch Affirmation, Subversion, Emphase oder Enthaltung. Die zwischen beiden Polen (Pop und x) entstehenden Wechselwirkungen lassen sich als ein »diskursives Feld widerstrebender Kräfte« (Goer/Greif/Jacke 2010: 212) beschreiben, das sich in der thematischen Breite der versammelten Artikel widerspiegeln soll. Pops Funktion kommt der eines gesellschaftlichen »Seismograph[en] (Pethes 2012: 108) gleich, der Bewegungen jeglicher Art aufzeichnet, abspeichert und verwertet: Medien, Musik, Mode, Politik, Trends, Idole, Lifestyle. Das bekannte Rainald-Goetz-Zitat, welches das Definitionsproblem an den Definierenden delegiert, eignet sich in diesem Sinne, um dem antiautoritären Terminus >Pop< und seiner Praxis den besagten Rahmen zu geben, ohne allzu feste Grenzen zu setzen:

»Pops Glück ist, dass Pop kein Problem hat. Deshalb kann man Pop nicht denken, nicht kritisieren, nicht analytisch schreiben, sondern Pop ist Pop leben, fasziniert betrachten, besessen studieren, maximal materialreich erzählen, feiern. Es gibt keine andere vernünftige Weise über Pop zu reden, als hingerissen auf das Hinreißende zu zeigen, hey, super. 
Deshalb wirft Pop Probleme auf, für den denkenden Menschen, die aber Probleme des Denkens sind, nicht des Pop.«(Goetz 2003: 188)

Wie lässt sich diese lapidar formulierte Deskription eines Popliteraten mit einem wissenschaftlichen Bandkonzept vereinen? Obwohl oftmals die Behauptung im Raum steht, Pop als eine >Kultur des aktiven Handelns an Kultur< sei nicht in ein statisches, wissenschaftliches Korsett zu zwängen und wenn doch, wäre es zugleich der Beweis dafür, dass Pop nun passé sei, können beide - Pop und Wissenschaft - produktiv zusammengebracht werden, indem das Spielerische, Schillernde, Ausufernde des Pop im theoretischen Diskurs als eben solches verhandelt und abgebildet wird. Und zwar >maximal materialreich <. Dazu bietet sich die Informations- und Formatfülle des Leitmediums Fernsehen (Shows, Serien, Soaps, Dokumentationen etc.) geradezu an. ${ }^{1}$ So wird es im Folgenden als wichtiger Distributionskanal der Popkultur in den Fokus gerückt, um u.a. zu erörtern, welche Strategien die Akteure der Popkultur im TV verfolgen, um massenwirksam zu agieren oder eben um subversiv festgefahrene Strukturen zu sprengen, welche Anleihen das Fernsehen selbst bei popästhetischen Verfahren tätigt, welche popkulturellen Entwicklungen im TV-Programm aktuell noch ausgespart werden oder wie das Prinzip der Serialität auf der einen Seite genutzt wird, um Zuschauer zu binden, auf der anderen Seite, um durch Brechungen oder Mehrdeutigkeiten die Eigenständigkeit des Publikums herauszufordern (vgl. Hickethier 2001: 12 u. 208).

Setzt man sich mit dem Pop-Phänomen auseinander, bleiben zwei mögliche Reaktionen, zwischen denen entschieden werden muss. Erstens: vor den ausufernden Grenzen und der schweren Erfassbarkeit des Begriffs zu kapitulieren. Oder zweitens: sich auf das keineswegs immer oberflächliche Spiel mit dem Alltäglichen einzulassen. Die Herausgeber und Autoren dieses Bandes haben sich für Letzteres entschieden, um die Reflexion des Fernsehens aus popästhetischer Perspektive voranzutreiben und erste Überlegungen anzustellen, wie Pop- und Fernsehforschung zusammengedacht werden können.

\section{Forschungsstand}

Jeweils für sich genommen, ist die Forschung zu Pop und Fernsehen heutzutage kaum noch zu überblicken. Programmstrukturen, Sendeanstalten, einzelne Formate oder Möglichkeiten medialer Adressatenbindung scheinen ebenso vielfältig

1 Vgl. hierzu weiterführend Hickethier (2001). 
erforscht wie die künstlerischen Ausdrucksformen des Pop, seine sozialen Facetten und historischen Dimensionen. Ein Blick auf die Publikationsgeschichte verrät indes, dass die wissenschaftliche Konjunktur beider Untersuchungsgegenstände divergiert. So erscheinen viele grundlegende Studien zum älteren Leitmedium >Fernsehen $<$ zwischen den späten 1970er Jahren und der Jahrtausendwende. Von einer akademisch breiteren Popforschung kann hierzulande dagegen erst seit wenigen Jahren gesprochen werden. ${ }^{2}$ Analog dazu stehen Untersuchungen zur Präsenz des Pop im Fernsehen oder zur Auseinandersetzung vieler PopKünstler mit dem Medium noch aus. Derzeit scheint es jedoch, als wollten die Sachwalter beider kultureller Felder keine Kenntnis voneinander nehmen.

Von dieser Abstinenz ausgenommen ist jene Clipkultur, die sich beim Fernsehpublikum seit Beginn der 1980er Jahre großer Beliebtheit erfreut und die Sehgewohnheiten vieler Zuschauer ebenso nachhaltig verändert wie die Vermarktung einzelner Interpreten und Songs. Zugespitzt formuliert haben sich bislang praktisch alle kultur- und sozialwissenschaftlichen Fachdisziplinen mit dem Phänomen >Musikvideo < beschäftigt und es sowohl intermedial als auch pädagogisch, wahrnehmungsästhetisch, ökonomisch oder genderrepräsentativ untersucht. Bereits die (Untertitel-)Titel einiger Abhandlungen - Musikvideos zwischen Avantgarde und Populärkultur (Hausheer/Schönholzer 1994), Videoclips und Visualisierung von E-Musik (Rötter 2000) - verraten indes, warum das Genre mehr Beachtung gefunden hat als andere Popeinflüsse auf das Fernsehen: Aufgrund seiner Formatvielfalt und schnellen Schnitte rückt man den musikalischen $>$ Werbefilm $<$ in die Tradition internationaler Programmkunst oder reflektiert ihn als Erbe multimedialer >Gesamtkunstwerke< wie Oper und Vaudeville. Im Gegenzug wird die Selbstreflexivität eines »Mediums, das die Bedingungen der eigenen Produktion ständig thematisiert«, nur am Rande als ein generell popkonstitutives Charakteristikum gewürdigt (Bergermann 2003: 478).

Diese Marginalisierung einer fernsehspezifischen Popästhetik lässt sich auch in den spärlichen Untersuchungen zu Autoren wie Rainald Goetz oder Benjamin von Stuckrad-Barre beobachten, die nicht nur gelegentlich in Late-Night-Shows auftreten, sondern ihre eigenen TV-Formate entwickeln und realisieren (vgl. Binczek 2012). Eher allgemein ist hier von Literatur im Fernsehen die Rede, wohingegen die medienkritischen Ursprünge und popästhetischen Zielsetzungen beispielsweise jener drei Folgen, die Goetz 2001 für das ZDF-nachtstudio kon-

2 Dass die Kunstgeschichte sehr früh schon produktiv und ohne hochkulturellen Dünkel auf die Pop Art reagiert, sei der Vollständigkeit halber an dieser Stelle erwähnt. Erinnert sei an Max Imdahls wegweisende Studie »Probleme der Pop Art«, die 1968 im Ausstellungskatalog zur vierten documenta erscheint (vgl. Imdahl 2013). 
zipierte, unerwähnt bleiben. Umgekehrt gilt seit Jahren als ausgemacht, dass sich Popliteratur bereits in den späten 1960er Jahren an Narrationstechniken und Rezeptionsweisen des Fernsehens orientiert. ${ }^{3}$ Insofern muss die Frage erlaubt sein, warum die mediale, aber eben ins Popästhetische gewendete Kompetenz im TV präsenter Autoren für die Untersuchung ihrer Selbstinszenierungen und Fernsehproduktionen unberücksichtigt bleiben soll.

Dass die Popmusik im Fernsehen viel umfassender erforscht wird und hier auch richtungsweisende Theoriedebatten über die Gemeinsamkeiten respektive Unterschiede von Pop- und Popularkultur geführt werden (vgl. Jacke 2009), mag erklären, warum popaffine Spartensender wie MTV oder VIVA und ihre kaum zu unterschätzende Rolle bei der (Pop-)Sozialisierung junger Fernsehzuschauer ${ }^{4}$ eher im wissenschaftlichen Fokus standen als ihre historischen Vorläufer bei den öffentlich-rechtlichen Rundfunkanstalten. Dabei sollte nicht übersehen werden, welchen Einfluss bereits Sendungen wie der Beat Club (1965-1972) oder Ilja Richters disco (1971-1982) auf die Verbreitung popkultureller Lebensstile und Moden nehmen. Gleichzeitig lenken sie die selektive öffentliche Wahrnehmung popästhetischer Entwicklungen oder poppolitischer Widerstandspotenziale. Denn das Fernsehen rezipiert Pop traditionell als Bestandteil der Jugendkultur, doch nicht jeder Musiker, Künstler oder Literat adressiert seine Werke an ein jüngeres Publikum. Darüber hinaus setzen sich einzelne Kunstszenen (Social Beat, Poetry Slam) von Anfang an aus verschiedenen Altersklassen zusammen. Andere Gruppierungen oder Subkulturen des Pop, man denke an HipHop oder Techno, erweisen sich als erstaunlich langlebig und entwickeln sich auch deshalb weiter, weil unter sämtlichen Insidern noch immer das >Wir-Gefühl< dominiert. Differenzierter betrachtet ist Pop also nicht deckungsgleich mit seiner Inszenierung im Fernsehen. Aber ihr verdankt sich die - mit Blick auf alle partizipierenden Künste und gesellschaftlich beteiligten Gruppierungen einseitige Popularität des Pop, dessen Geschichte inzwischen auch fernsehseitig aufgearbeitet wird.

Zum aktuellen Forschungstrend, dem sich auch mehrere Abhandlungen des vorliegenden Bandes anschließen, gehört die Beschäftigung mit den Selbstinszenierungen der Dichter in den Medien und die Fremdinszenierung des Autors durch Malerei, Fotografie, Radio oder das Fernsehen selbst. Erstaunlicherweise spielt das TV in den bisher vorliegenden Untersuchungen zum Thema allerdings nur eine untergeordnete Rolle - gleichsam so, als gebe es nicht die lange Tradi-

3 Vgl. Ernst (2001) u. Greif (2004).

4 Vgl. Neumann-Braun (1999), Fritzsche (2003) u. Kaiser (2007). 
tion der Literaturmagazine, Autorendokus oder Dichter in Talkshows. ${ }^{5}$ Kritischer mit den Rezeptionsbedingungen des Fernsehens setzt sich derzeit der fernsehmedial weitgehend ignorierte Post- und Popfeminismus auseinander. Angeregt durch die Gendertheorie Judith Butlers, wird die mediale Repräsentation von Frauen entweder zum Gegenstand politischer Diskurse, oder Popfeministinnen erörtern am Beispiel des seriellen Erzählens in amerikanischen Fernsehproduktionen eine autarke Do-it-Yourself-Kultur. ${ }^{6}$

\section{Artikelteaser}

Dass die einzelnen Artikel eines Sammelbands auch selbstständig funktionieren und überzeugen, setzt man geläufig voraus. Erst deren Konstellation generiert jedoch den >Mehrwert<, der im besten Fall über die Summe der einzelnen Teile hinausgeht und dem Band Kontur verleiht. Um das breite Spektrum an Sujets, Popbegriffen und Herangehensweisen rund um Popkultur und Fernsehen zu systematisieren, schien uns die folgende Dreiteilung für das skizzierte Konzept naheliegend.

\section{Pop|TV|Ästhetik}

Mit der Anordnung der Artikel innerhalb dieses Bedeutungshorizonts soll das Versprechen des Banduntertitels eingelöst werden: Das Kapitel gibt einen schlaglichtartigen Überblick wichtiger Pop und TV zusammenführender Traditionslinien, sodass die ästhetischen Berührungspunkte (etwa Serialisierung, mediale Oberflächen, Popularisierung, Starkult etc.) historisch lesbar werden als (sehr knappe) Pop- und TV-Geschichte von den Anfängen bis zum >medienkonvergenten Gegenwartsfernsehen $<$. Ein besonderer und immer wiederkehrender Akzent liegt dabei auf der Rolle des Rezipienten: Als Nebeneffekt lässt sich dessen Entwicklung auch als Historie der Aktivierung und Selbstbestimmung lesen, obwohl sich dieser Weg in der Gegenwart tendenziell zu gabeln scheint.

Helmut Schanze zeichnet in » The Most Popular Art < - Popkultur, Fernsehen und die Anfänge der Medienwissenschaft« diejenigen epistemologischen Linien, die im Fluchtpunkt der 1950er Jahre zusammenlaufen, in den Popdiskurs ein. Kunst, Medien, Ästhetik und Publikum sind die Faktoren, die Schanze nicht als separate, sondern vielmehr als im Stichwort der Popularität konvergierende ver-

5 Vgl. Künzel/Schönert (2007) u. Jürgensen/Kaiser (2011).

6 Vgl. Lenzhofer (2006) u. Köver (2007). 
standen wissen will. Sein detailreicher und geschichtsträchtiger Beitrag, der mit besonderer Berücksichtigung der Jahre um 1970 Pop und TV zusammendenkt, datiert dabei die Wurzeln heutiger Popkultur bis ins späte achte Jahrhundert zurück. Bereits damals entbrannte nämlich der Streit um Popularität und Trivialität, um die Frage, ob die >Vox populi<, die Stimme des Volkes, eher >göttlich oder >wahnsinnig $<$ genannt zu werden verdiene.

RALF SCHNELL lotet ganz in diesem mediengeschichtlichen Sinne diejenigen popästhetischen Mechanismen aus, mittels derer der Spiegel bereits in den 1950er Jahren Formen der textuellen und visuellen Autoreninszenierung entwickelt, die dann vom Fernsehen in einer strukturellen Analogie aufgegriffen wurden und das öffentliche >Bild $<$ des lebenden oder toten Autors nachhaltig veränderten. Sein Beitrag »Cherchez la femme!< - Anmerkungen zur literarischen Ikonografie des frühen Spiegel und zur Popularisierung literarischer Hochkultur im jungen deutschen Fernsehen « unterstreicht dabei am Beispiel Ingeborg Bachmanns die Verbindung von Literaturbetrieb und Popkultur zur >Kulturpopularisierung $<$, die der Spiegel dem >anspruchsvollen $<$ jungen deutschen Fernsehen der 50er und 60er vorgelebt hatte.

In den 60er, 70er und 80er Jahren ist Andy Warhol vor und hinter der Kamera tätig. STEFAN GREIF führt den Nachweis, dass Warhols popästhetische TVArtefakte zu Unrecht als Armutszeugnis eindimensionaler Egozentrik rezipiert worden sind, geben sie doch in ihrer stets metamedialen Reflektiertheit vielmehr Zeugnis davon, wie der Rezipient gerade mit den Mitteln popästhetischer Verfahren eine massive Aufwertung erfährt, die ihm die sogenannte Hochkultur versagt. Am Beispiel besagter Fernseharbeiten zeigt Greif in seinem Artikel »15 Minutes of Fame - Andy Warhol als Superstar seines Popkosmos « auf, wie der Starkult im Fernsehen und mit ihm der >Totalitarismus $<$ der Moderne vom >Godfather of Pop< ad absurdum geführt und die Rezipienten medial zu ihrer eigenen $>$ Subjektwerdung $<$ angeleitet werden.

Den Versuch, die Kunst aus den >heiligen Hallen< des Museums herauszuholen und die Grenzen zwischen aktivem Aktionsraum und passivem Publikum zu verwischen, treten Happening und Fluxus in den 1960er Jahren an. Die damit einhergehende Überzeugung, dass Kunst diskursiv sei, der Zuschauer, statt festgeschriebenen und unter der Deutungshoheit kleiner Eliten stehenden Kardinalinterpretationen zu gehorchen, zur Partizipation eingeladen ist, betont auch in diesem Beitrag den zentralen Stellenwert des Rezipienten. Mit einer der Wegmarken, nämlich der Ausstellung Happening \& Fluxus (Köln, 1970), beschäftigt sich KAI-Uwe HEMKEN in seinem Kunsthistorie, -theorie, und -ästhetik vereinenden Aufsatz »Expressive Subjekte vor der Kamera - Zur Relativität von Kunst, Ausstellung und Massenmedien um 1970«. Mit einer Fokussierung auf 
die TV-Reportage zur Ausstellung beleuchtet Hemken neben dem >Verhältnis von Masse und Elite< exemplarisch die fernsehästhetische Dimension von $>$ Kunst und Aufmerksamkeitsregime < in der >mediatisierten Öffentlichkeit<.

In zweierlei Hinsicht ist die schillernde Figur Christoph Schlingensief als >Apologet< dieser Avantgarde-Entwicklung zu sehen: Einerseits gilt er selbst als Fluxuskünstler, andererseits zeichnet Schlingensief als Multiplikator dafür verantwortlich, performative Verfahrensweisen auch in die Fernsehkunst überführt zu haben. In »Performativität und Format - Anmerkungen zu Christoph Schlingensiefs Fernseharbeiten« stellt BERND MAUBACH Thesen zu dessen popästhetischen Aktionen im TV auf. Im angegriffenen Medium selbst ausgestrahlt, erregten sie als subversive Formatkritik großes Aufsehen, konnten sich doch weder Akteure noch Zuschauer >adäquat< verhalten. Denn beide waren häufig gezwungen, zwischen zwei zur Auswahl stehenden Wahrnehmungs- und Deutungsrahmen zu wählen, die jeweils nicht hundertprozentig situationsangemessen sind. Dadurch entsteht der Eindruck einer Prozesskunst-Ästhetik zwischen Reizüberladung und Anarchie, welche sich als Höhepunkt der Rezipientenaktivierung, im Fall Schlingensiefs manchmal aber auch als grenzüberschreitender Schritt zur hoffnungslosen Publikumsüberforderung verstehen lässt.

Als alternative Konsequenz dieser Entwicklung lässt sich etwa die heutige Scripted-Reality-Soap Berlin - Tag \& Nacht, ein gerade unter Jugendlichen sehr populäres Format, werten. An deren Modell geht ACHIM BARSCH in seiner Untersuchung »TV-Marken als Strategien in der Popularkultur - Das Beispiel Berlin - Tag \& Nacht« der Frage nach, wie TV-Sender verschiedene Medien in ihrer Konvergenz nutzen und diverse Instrumente entwickeln, um zu einer über den Sender hinausgehenden festen Zuschauerbindung zu gelangen. Mit den Schlagworten >Partizipation $<$, > Transformation $<$ und $>$ Durchdringung $<$ umreißt er nicht nur Marketingstrategien und eine popkulturelle Fernsehästhetik der Jetztzeit, sondern schreibt auch die Pop- und TV-Geschichte des aktivierten und medial ausdifferenzierten, dabei in zunehmendem Maße aber gleichzeitig konfektionierten Fernsehpublikums fort.

\section{Pop|Kultur|Akteure}

Zusammen mit der ästhetischen Kategorie der Authentizität verabschiedete die Forschung schon vor geraumer Zeit die Vorstellung, ein medial vermitteltes Bild des Schriftstellers, Musikers oder Regisseurs könne mit der lebenden Person identisch sein. Andererseits entdeckte man den >lebenden Menschen< hinter den Konstrukten der Dekonstruktivisten neu. Beides in eins nehmend, gehen die Beiträge dieses Kapitels den Aspekten von medialen Selbst- und Fremdinszenierungen einzelner Akteure der Pop- und Populärkultur im Massenmedium Fernsehen 
nach. Trotz der unterschiedlichen Gewichtung der Artikel wird als Vergleichsgrundlage immer wieder darauf rekurriert, welche Attribuierungen gewählt werden, in welchem Rahmen dies geschieht und was für ein Erfolg, gemessen an der jeweiligen Zielsetzung, solchen Konstruktionen beschieden ist.

PETER SEIBERT beschäftigt sich in seinem Beitrag »Autoren im Dschungelcamp oder: >Warum habe ich weniger Minuten als der Pornostar? - Anmerkungen zur Fernsehinszenierung von Autoren « damit, wie prinzipiell das Fernsehen mit seinen medienspezifisch-popästhetischen Potenzialen für die (Selbst-)Präsentation von Autoren einerseits, für die Aushandlung verschiedener Konzepte von $>\mathrm{Au}-$ torschaft< andererseits genutzt werden kann und genutzt wird. Der Artikel untersucht aber nicht nur die affirmativen oder subversiven populären Selbstinszenierungen der Autoren im Fernsehen, sondern auch, wie und warum selbst medienkompetente Pop-Autoren der Fremdinszenierung bzw. >Okkupation< durch das TV unterliegen. Damit scheinen die Spielräume, um die Muster von Autorenzuschreibungen und die diesbezügliche Deutungshoheit des Fernsehens zu unterlaufen, äußerst gering.

Christian Kracht ist einer jener mediensozialisierten und medienversierten Pop-Akteure. Seine Inszenierungsstrategien im TV, die zwischen Ironie und totaler Informations- bzw. Kommunikationsverweigerung oszillieren, entdeckt NILS LEHNERT anhand der unterschiedlich gerahmten Sendeformate druckfrisch und der Harald Schmidt Show. Die These seines Aufsatzes »Refus aus Kalkül?! $\mathrm{Zu}$ Christian Krachts Fernsehauftritten « besagt, dass bei allem (gerade auch wissenschaftlichen) Goutieren der >lauten< Selbstdarstellungsstrategien von Popautoren, die >leisen<, subtileren Verfahren gelegentlich ins Hintertreffen der Aufmerksamkeit geraten, obzwar gerade sie es sind, die subversiv Sand ins Getriebe der Medienmaschine streuen können. Qua höflicher Zurückhaltung, Passivität und Refus glückt Krachts > Ästhetik des Verblassensく, womit er sich selbst un(an)greifbar macht.

JULIA PATER macht in »)Oder ist das Fernsehen schon wirklicher als wie die Wirklichkeit? - Rainald Goetz' popästhetische Inszenierung im Fernsehen « die mediale Selbstdarstellung zum Analyseobjekt ihrer Untersuchung. Insbesondere in Late-Night-Shows, aber auch in literarischen Fernsehmagazinen inszeniert sich Goetz auf irritierende und mitunter skurril anmutende Art und Weise. Obwohl die performative Komponente und das Schockante mit der Zeit abnehmen, sorgen seine Auftritte (und auch sein Wirken als Fernsehproduzent) immer wieder für Verwirrung. Ob und wie sich diese Beobachtungen mit der bewussten Subversion der vom Pop-Autor selbst missbilligten Redundanz des Massenme- 
diums TV vertragen, diskutiert der Beitrag im Kontext der Strategien medialer Autorenselbstinszenierungen.

Alfonso MeOli richtet den Analysefokus in seinem Beitrag »Guru. Superstar. Nickelbrillen-Esoteriker. - Über die Hermann-Hesse-Konstruktionen in den Fernsehportraits zum 50. Todestag « auf die Produktionsseite, also darauf, welche Fremdinszenierungen die jeweiligen Portraits einsetzen, um zwei unterschiedliche Hesse-Bilder vorzulegen und diese für zwei unterschiedliche Publika attraktiv zu machen. Dabei wird diskutiert, ob die popkulturellen Techniken sich anverwandelnde Regiehandschrift Andreas Ammers mittels Sampling wirklich Neues schöpft oder vielmehr eine mediale Materialcollage ist. In jedem Fall weist Meoli nach, dass Hesse von ein und demselben Sender (SWR) im Gedenkjahr auf vollends unterschiedliche Weise konstruiert wird - einmal als Einsiedler verklärt, einmal als Popstar verehrt.

Lässt sich auch der historische >Star< Wolfgang Amadeus Mozart mit den Mitteln popästhetischer Verfahren heutigen jungen Zuschauern schmackhaft machen? Und welcher Art müsste diese mediale Fremdinszenierung dann gestaltet sein? ANDREAS WICKE wendet sich in seinem Aufsatz »>wegen dem sogenannten Popolare sorgen sie nichts $<$ - Zum Mozart-Bild in der Animationsserie Little Amadeus « diesen Fragen zu und beleuchtet im Abgleich mit anderen medialen Mozart-Konstruktionen darüber hinaus, welche Rollen dabei die musikalische und historische Wissensvermittlung einnehmen und welche Berührungspunkte popkultureller und fernsehästhetischer Machart existieren. Schließlich zeigt Wicke auf, dass bereits im 18. Jahrhundert moderne popkulturelle Losungen wie >Marktorientierung < und >Mehrfachadressierung < keine Fremdworte gewesen sind.

\section{TV $\mid$ Genre|Pop}

In der dreifachen Lesart >TV-Genre: Pop $<, ~>T V$, Genre, Pop $<$ und $>$ TV und Genre-Pop< subsumiert dieses Kapitel vier Artikel zu aktuellen Entwicklungen und Genres der Popkultur in Theorie und Fernsehpraxis sowie - andersherum gedacht - die popkulturanalytische Sicht auf das Fernsehen aus der Perspektive benachbarter Pop-Genres (Literatur, Musik, Poetry Slam). Es geht also um die Macht der Television, Genres und ganze Szenen zu formatieren, aber auch um die Macht des Pop, das Fernsehen und dessen popaffine Genres kritisch zu reflektieren. Als Gemeinsamkeit teilen die Beiträge die Einsicht, dass die dem Pop schon immer innewohnende Relativierung von unterhaltenden und ernsten Anteilen, von Mainstream und Gegenöffentlichkeit für die Verbindung von Fernsehen und Popkultur stets neu gedacht und neu geschrieben werden muss. 
Andreas Neumeister stellt, wie AnNA-CARINA MeYwiRTH in ihrem Aufsatz »Die wirkliche Welt auf wirklichen Bildschirmen $<$ Fernsehen in Andreas Neumeisters Angela Davis löscht ihre Website « belegt, Medieninhalte und deren vermeintliche Wirklichkeitsabbildungen infrage, indem er die konstruierte Bildschirm-Realität des Fernsehens durch Montage zerstört und damit eine ganz neue Dimension der Wechselwirkung von (Pop-)Literatur und TV eröffnet. > Fiction $<$ und >non-fiction $<$, $>E$ und $\mathrm{U}<$, >Realität< und $>$ Fernsehrealität $<$ verlieren in Neumeisters Textcollage nämlich ihre Trennschärfe und ihre Orientierungsfunktion. Nicht linear, sondern assoziativ sind seine Textbruchstücke infolgedessen konzipiert, um den Leser durch diesen popästhetischen Komplexitätsgewinn zu >entautomatisieren< (Weingart) und so einer passiven Rezeption zuvorzukommen.

Dass bestimmte (gegenöffentliche) Genres popaffine Züge tragen, das Fernsehen seine (Rezeptions-)Spuren in ihnen hinterlässt und sie wiederum auf das Fernsehen abfärben, gilt als ausgemacht. Mit Ausnahme von Kulturformaten, wie der Sendung Tracks auf Arte, oder popfeministisch lesbaren Serien, wie etwa der HBO-Produktion Girls, wird indes der Popfeminismus im TV kaum repräsentiert, obwohl das Thema in Magazinen, Büchern, Podcasts und Blogs hochaktuell ist. In ihrer Analyse » TV-Glotzer $<$ - Überlegungen zu Popfeminismus und Fernsehen « benennt ANNA SEIDEL bisher verbliebene Leerstellen und untersucht neben der Präsenz des Popfeminismus in verschiedenen TV-Genres auch dessen prinzipielle Verquickung mit dem Medium Fernsehen etwa in der popfeministischen Kulturkritik.

Mit einem ebenso aktuell umforschten Thema setzt sich MAX DORN auseinander: Er untersucht in seinem Beitrag »>Man füllt also irgendwie fünf Minuten Bühnenzeit<. Zur Entwicklung des Poetry Slams unter dem Einfluss des Fernsehens im deutschsprachigen Raum « die Wechselwirkungen von Poetry Slam, Slam Poetry und TV in Zeiten, in denen ein >alterndes $<$ Medium jüngeren Popphänomenen eine neue Bühne bereitet. Im Zentrum der Aufmerksamkeit steht die veränderte Wahrnehmung des Zuschauers, sobald mediale Filter und eine Doppeladressierung auf den Plan treten. Welch einschneidende und mainstreamwirksame Folgen das Fernsehen für die zunächst wenig telegene Gattung der performativen Dichtung zeitigte, die sich als soziale und politische in den 1980er Jahren im Off konstituierte, zeigt Dorn historisch und ästhetisch gleichermaßen.

Musiksender wie MTV und VIVA haben popmusikalischen Themen in Form von Videoclips, Musik-Talks oder Dokumentationen seit Anfang der 1980er Jahre einen Platz in den bewegten Bildern gesichert. Aktuell werden sie ergänzt durch Onlineformate wie PutPat.tv oder tape.tv. Aber wie ist es im öffentlichrechtlichen Fernsehen um die Popmusik bestellt? In ihrem Aufsatz »Popmusik 
und Fernsehen - Exemplarische Untersuchung öffentlich-rechtlicher Fernsehsender und popmusikalischer Inhalte nimmt KRISTINA FLIEGER in Form von qualitativen Fallanalysen die Inhaltsprofile öffentlich-rechtlicher Fernsehsender unter die Lupe, um Quantität und Qualität popmusikalischer Formate zu bestimmen. Dadurch kann sie eindeutig nachweisen, dass die immer wieder beklagte Entwicklungsrichtung der Trivialisierung zumindest für das Genre der Popmusik in dieser Verknappung nicht haltbar ist.

Abgerundet wird der Band mit dem Perspektivwechsel von der theoretischen zur produzierend-praktischen Sicht auf Pop und TV mit dem eigens für diesen Band geführten Interview »Zur Not auch die ZDF-Hitparade. Ein Gespräch über Popmusik und Fernsehen in Deutschland «. Darin erörtern MARKUS KAVKA und Christoph JACKE die Geschichte des Popmusikfernsehens seit den 1980er Jahren und gehen gleichzeitig der Frage nach, warum diese Programmsparte heute wieder zu den Nischen- oder Liebhaberformaten gehört. Darüber hinaus eröffnet Markus Kavka wertvolle Einblicke in das Selbstverständnis ehedem erfolgreicher Jugendsender wie MTV oder VIVA, denen es mit Ende der 1990er Jahre nicht gelingt, die Konkurrenz mit den Neuen Medien als Chance zu begreifen.

$* * *$

Dem vorliegenden Buch voran ging die Tagung Pop- und Populärkultur im Fernsehen, die am 05. und 06. Februar 2014 anlässlich des fünfundsechzigsten Geburtstags von Peter Seibert an der Universität Kassel stattfand. Aus den anregenden Vorträgen und Diskussionen entwickelte sich die Idee, nicht nur einen Tagungsband zu veröffentlichen, sondern darüber hinaus Aufsätze aus dem interdisziplinären Mitarbeiterumfeld des Jubilars und aus dem Kreis seiner Studierenden einzuwerben, die sich aktuell mit dem breitgefächerten Thema Pop und $T V$ beschäftigen.

Besonderer Dank gebührt an dieser Stelle Katharina Zindel und Max Dorn, die in vielen Tag- und Nachtschichten die elektronische Einrichtung sämtlicher Beiträge übernommen haben. ${ }^{7}$

7 Mit Nennung der weiblichen [männlichen] Funktionsbezeichnung ist in diesem Buch, sofern nicht anders gekennzeichnet, immer auch die männliche [weibliche] Form mitgemeint. 


\section{Literatur}

Baßler, Moritz (2002): Der deutsche Pop-Roman. Die neuen Archivisten, München: C.H. Beck.

Bergermann, Ulrike (2003): »Videoclip«, in: Hans-Otto Hügel (Hg.), Handbuch Populäre Kultur. Begriffe, Theorien und Diskussionen, Stuttgart/Weimar: Metzler, S. 478-482.

Binczek, Natalie (2012): »Fernsehauftritte der Literatur: Rainald Goetz«, in: Sprache und Literatur 109, S. 73-88.

Diederichsen, Diedrich (1996): »Pop - deskriptiv, normativ, emphatisch«, in: Marcel Hartges (Hg.), Pop, Technik, Poesie: Die nächste Generation (= Literaturmagazin, Band 37), Reinbek bei Hamburg: Rowohlt, S. 36-44.

Ernst, Thomas (2001): Popliteratur, Hamburg: Europäische Verlagsanstalt.

Fritzsche, Bettina (2003): Pop-Fans. Studie einer Mädchenkultur, Opladen: VS.

Goer, Charis/Greif, Stefan/Jacke, Christoph (2010): »Poptheorie, Popkulturforschung und Literaturwissenschaft«, in: Lothar van Laak (Hg.), Literaturwissenschaft - interdisziplinär, Heidelberg: Synchron, S. 211-230.

Dies. (Hg.) (2013): Texte zur Theorie des Pop, Stuttgart: Reclam.

Goetz, Rainald (2003): »Und Blut«, in: Ders., Hirn, Frankfurt am Main: Suhrkamp.

Greif, Stefan (2004): »In Video veritas. Rainald Goetz' videographische Fernsehdokumentation 1989«, in: Felix Holtschoppen/Frank Linden/Friederike Sinning et al. (Hg.), Clips. Eine Collage, Münster: LIT, S. 115-132.

Hausheer, Cecilia/Schönholzer, Annette (Hg.) (1994): Visueller Sound. Musikvideos zwischen Avantgarde und Populärkultur, Luzern: Zyklop.

Hecken, Thomas (2009): Pop: Geschichte eines Konzepts 1955-2009, Bielefeld: transcript.

Ders. (2012): »Pop: Aktuelle Definitionen und Sprachgebrauch «, in: PopZeitschrift vom 08.09.2012. Online unter: www.pop-zeitschrift.de/2012/09/ 09/pop-aktuelle-definitionen-und-sprachgebrauchvon-thomas-hecken/

Hickethier, Knut (2001): Film- und Fernsehanalyse, Stuttgart: Metzler.

Imdahl, Max (2013): »Probleme der Pop Art«, in: Charis Goer/Stefan Greif/ Christoph Jacke (Hg.), Texte zur Theorie des Pop, Stuttgart: Reclam, S. 6175.

Jacke, Christoph (2009): Einführung Populäre Musik und Medien, Münster u.a.: LIT.

Jürgensen, Christoph/Kaiser, Gerhard (Hg.) (2011): Schriftstellerische Inszenierungspraktiken: Typologie und Geschichte, Heidelberg: Winter. 
Kaiser, Sabine (2007): Gespräche über Fernsehen. Die Bedeutung der nachrezeptiven Phase zur Konstitution von Geschlechtsidentität im Jugendalter, Bielefeld: Universitätsverlag.

Köver, Chris (2007): »Couch-Politik. Wo bei TV-Serien in puncto Feminismus was zu holen ist«, in: Sonja Eismann (Hg.), Hot Topic. Popfeminismus heute, Mainz: Ventil, S. 134-140.

Künzel, Christine/Schönert, Jörg (Hg.) (2007): Autorinszenierungen: Autorschaft und literarisches Werk im Kontext der Medien, Würzburg: Königshausen \& Neumann.

Lenzhofer, Karin (2006): Chicks Rule! Die schönen neuen Heldinnen in USamerikanischen Fernsehserien, Bielefeld: transcript.

Neumann-Braun, Klaus (Hg.) (1999): Viva MTV! Popmusik im Fernsehen, Frankfurt am Main: Suhrkamp.

Pethes, Nicolas (2013): »Pop-Wissen«, in: Pop. Kultur und Kritik 3, S. 102-112.

Rötter, Günther (2000): »Videoclips und Visualisierung von E-Musik«, in: Josef Kloppenburg (Hg.), Musik multimedial. Filmmusik, Videoclip, Fernsehen, Laaber: Laaber-Verlag, S. 259-294.

Steenblock, Volker (2010): »Bemerkungen zur Popkultur«, in: Thomas Hecken/Marcel Wrzesinski (Hg.), Philosophie und Popkultur (= Schriften zur Popkultur, Band 5), Bochum: Posth, S. 193-202. 
